\title{
Prenatal diagnosis of Fraser syndrome caused by novel variants of FREM2
}

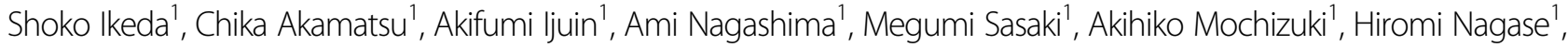 \\ Yumi Enomoto ${ }^{2}$, Yukiko Kuroda ${ }^{3}$, Kenji Kurosawa $\mathbb{0}^{3}$ and Hiroshi Ishikawa ${ }^{1}$
}

\begin{abstract}
Fraser syndrome (FS) involves multiple malformations and has a $25 \%$ recurrence risk among siblings. However, these malformations are difficult to detect prenatally, hampering prenatal diagnosis. Here, we describe a fetus with FS diagnosed using ultrasonography. Ultrasonography revealed congenital high airway obstruction syndrome and renal agenesis. Syndactyly of both hands and cryptophthalmos were noted postnatally, and the diagnosis was confirmed by genetic analysis, which showed novel compound heterozygous variants of FREM2.
\end{abstract}

Fraser syndrome (FS; cryptophthalmos-syndactyly syndrome; OMIM \#219000) is a rare autosomal recessive multiple malformation syndrome characterized by cryptophthalmos, syndactyly, and respiratory and urogenital tract anomalies ${ }^{1-4}$. Over 250 cases have been reported to date $^{2}$. The prevalence of FS is 0.43 per 100,000 and 11.06 per 100,000 among newborns and stillborns, respectively ${ }^{5}$. FS is a genetically heterogeneous condition, and pathogenic variants of FRAS1 and FREM2, which encode a family of large extracellular matrix (ECM) proteins, are implicated in the etiology of FS. These proteins are essential for the adhesion of epidermal basement membranes to dermal connective tissues during embryogenesis $^{6,7}$. Because anomalies such as fused eyelids and those in the digits, larynx, and vagina result from failed programmed cell death, defective apoptosis seems to be the key mechanism underlying FS. Recently, pathogenic variants of GRIP1, which encodes glutamate receptorinteracting protein 1 , have also been identified to cause FS in humans ${ }^{8}$.

\footnotetext{
Correspondence: Shoko Ikeda (shoko.i.1210@gmail.com) or

Kenji Kurosawa (kkurosawa@kcmc.jp)

'Department of Obstetrics and Gynecology, Kanagawa Children's Medical Center, Yokohama, Japan

${ }^{2}$ Clinical Research Institute, Kanagawa Children's Medical Center, Yokohama Japan

Full list of author information is available at the end of the article.
}

The current clinical diagnostic criteria proposed by van Haelst et al. include six major manifestations (syndactyly, cryptophthalmos spectrum, ambiguous genitalia, urinary tract abnormalities, laryngeal and tracheal anomalies, and positive family history) and five minor symptoms (anorectal defects, dysplastic ears, skull ossification defects, umbilical defects, and nasal anomalies) ${ }^{2}$. The authors suggested that a diagnosis of FS can be made if either three major, two major and two minor, or one major and three minor symptoms are present in a patient.

As FS is an autosomal recessive disease and has a recurrence risk of $25 \%$ among siblings, both prenatal and postnatal diagnoses are important in affected families. However, major manifestations, such as cryptophthalmos and syndactyly, are difficult to detect via prenatal ultrasonography, especially in the presence of oligohydramnios. Therefore, few prenatal cases have been reported.

Here, we report a case of FS prenatally suspected at 19 weeks of gestation and diagnosed via genetic analysis, which uncovered novel compound heterozygous FREM2 variants. This study was approved by the Institutional Review Board of Kanagawa Children's Medical Center, and clinical information was collected after obtaining written informed consent from the parents.

A 38-year-old female, gravida 2, para 0 , was referred to our hospital because of oligohydramnios and fetal ascites 


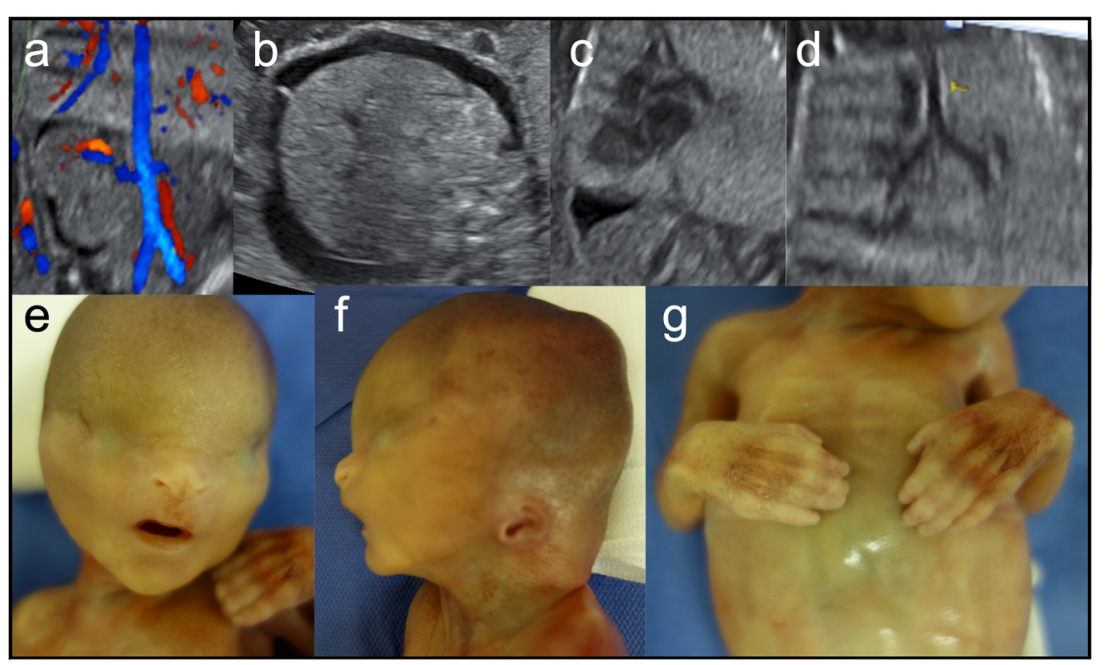

Fig. 1 Prenatal ultrasonography images and postnatal diagnosis of a fetus with Fraser syndrome. Bilateral renal agenesis: color Doppler image of the renal artery (a). Severe ascites (b), a hyperechogenic lung with an inverted diaphragm (c), and a distended trachea (d) were observed at 19 weeks of gestation. Fetal facial features included cryptophthalmos, hypertelorism, a small, low-set nose with a bifid tip, and microtia (e, f). Bilateral cutaneous syndactyly of the hands was also observed $(\mathbf{g})$.

at 19 weeks of gestation. The parental history of the fetus was unremarkable, and consanguinity was absent. Ultrasonography revealed severe oligohydramnios, bilateral renal agenesis, no urinary bladder, and numerous ascites (Fig. 1a, b). Head and long-bone biometry results were normal; however, the abdomen was distended owing to ascites and hepatomegaly. The lungs were enlarged and hyperechogenic, with an inverted diaphragm (Fig. 1c). The trachea was distended from the caudal of the carina to the bronchi (Fig. 1d). The heart and intracranial structures were normal. The digits of the hands and feet were barely visible. The enlarged lungs, distended trachea, and ascites indicated congenital high obstruction airway syndrome (CHAOS). The concurrence of CHAOS and renal agenesis prompted us to suspect FS, which met two major criteria $^{2}$. Given the poor prognosis, the parents terminated the pregnancy at 21 weeks. A male infant was delivered with a birth weight of $520 \mathrm{~g}$ and a length of $29.5 \mathrm{~cm}$. His facial features included bilateral cryptophthalmos and low-set, malformed ears (Fig. 1e, f). Syndactyly of both hands (I-IV) was also noted (Fig. 1g). Autopsy revealed pleural effusion and ascites, atresia of the epiglottis with a dilated trachea, hyperinflated and heavy lungs, and agenesis of the kidneys, ureters, and bladder. Chromosomal analysis revealed a normal male karyotype.

Genomic DNA was extracted from the umbilical cord blood of the fetus and peripheral blood of the parents using a QIAcube Kit (QIAGEN, Hilden, Germany). Targeted sequencing was performed using a TruSight One Sequencing Panel (Illumina, San Diego, CA, USA) on the MiSeq platform (Illumina) with 151-bp paired-

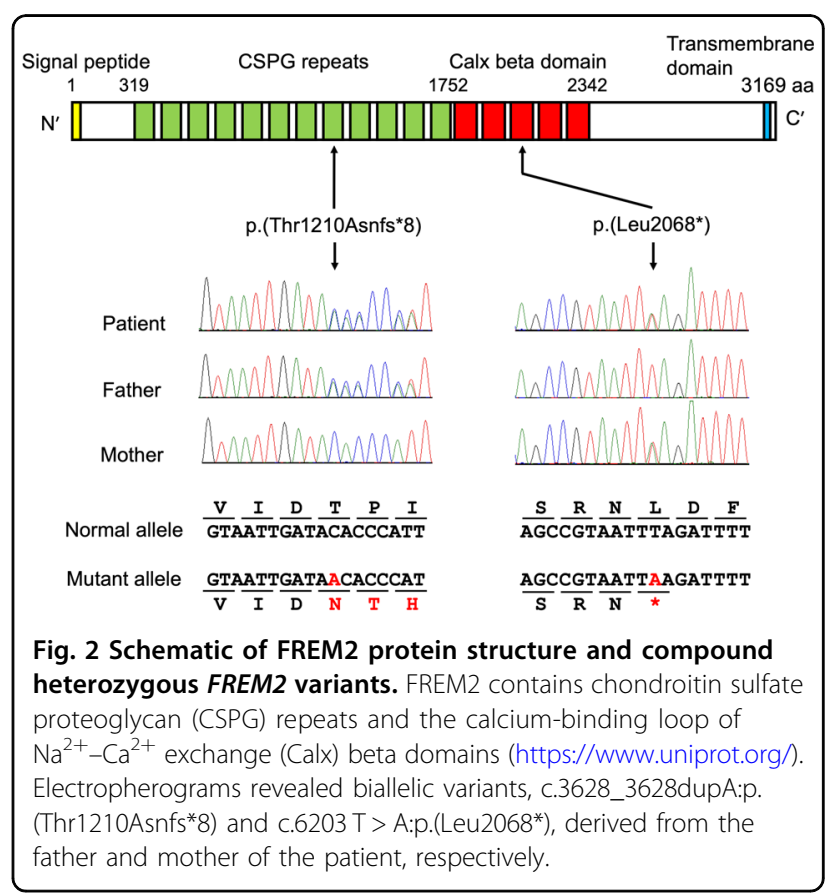

end reads, as previously described ${ }^{9}$. Candidate variants were subsequently confirmed by Sanger sequencing.

We identified the compound heterozygous variants: NM_207361.5:c.3628_3628dupA:p.(Thr1210Asnfs"8) and c.6203 T > A:p.(Leu2068*) of FREM2. Sanger sequencing confirmed that the c.3628_3628dupA variant was inherited from the father, whereas the c.6203 $\mathrm{T}>\mathrm{A}$ variant was inherited from the mother (Fig. 2). These variants are novel and absent in gnomAD 
(https://gnomad.broadinstitute.org/), ClinVar (https:// www.ncbi.nlm.nih.gov/clinvar/), jMorp (https://jmorp. megabank.tohoku.ac.jp/202001/variants), the Human Genetic Variation Database (http://www.hgvd.genome. med.kyoto-u.ac.jp/), and the Human Genome Mutation Database 2019.4 (https://portal.biobase-international. $\mathrm{com} /$ ). Both FREM2 variants are predicted to contain premature termination codons, consistent with biallelic loss-of-function.

FRAS1 mutations are the most common cause of FS, accounting for $65 \%$ of cases, while FREM2 mutations account for $20 \%$ of cases, and GRIP1 is associated with a limited number of cases ${ }^{8,10}$. Thus, definitive diagnosis is difficult in families without prior affected siblings. To date, only two prenatally diagnosed FS cases without any family history have been reported ${ }^{11,12}$. To our knowledge, this is the first report of a Japanese FS patient carrying FREM2 pathogenic variants.

FRAS1 and FREM2 are multidomain ECM transmembrane proteins, and GRIP1 is required for the presentation of the FRAS/FREM complex at the basal membrane of epithelial cells. ECM proteins are important for cell adhesion and migration, basement membrane integrity, and epithelial-mesenchymal interactions during development $^{6,7,13}$. Metanephric kidney development depends on interactions between the ureteric bud epithelium and adjacent mesenchyme, during which ECM proteins are involved ${ }^{14}$. Cryptophthalmos and syndactyly arise from the loss of epidermal adhesion, causing interrupted epidermal/mesenchymal interactions between the eyelid epithelia or limb apical ectodermal ridge and the underlying mesenchyme ${ }^{15}$.

Ultrasonography is an important tool for the prenatal diagnosis of malformations. Teisser et al. analyzed 38 pre- and postnatal cases (of which 36 had a family history) and observed bilateral or unilateral renal agenesis and airway obstruction in 61 and $42 \%$ of the fetuses, respectively, by ultrasonography ${ }^{3}$. The same authors observed eye anomalies, including microphthalmia or hypertelorism, and syndactyly in only 15 and $8 \%$ of the cases, respectively. Laryngeal atresia is a common cause of CHAOS and involves large echogenic lungs, a flattened or inverted diaphragm, dilated airways below the level of the obstruction, and fetal ascites or hydrops. Fetuses with CHAOS often exhibit polyhydramnios, but in FS, CHAOS with oligohydramnios can occur because of severe renal insufficiency ${ }^{16}$. Our patient was referred because of fetal ascites. To determine the cause, we investigated the fetus in detail using ultrasonography and discovered that laryngeal atresia was the cause of ascites. This satisfied two major criteria of FS, strongly suggesting this diagnosis. As in the present case, the concurrence of renal agenesis and laryngeal atresia is recognized as a valuable sonographic marker of FS, especially in patients without affected siblings ${ }^{3,10,17,18}$. In routine ultrasound examinations, the coexistence of fetal hydrops (including isolated ascites) and oligohydramnios seems to indicate a differential diagnosis of FS. Additionally, syndactyly, abnormal genitalia or dysplastic ears may facilitate the diagnosis of FS via ultrasonography ${ }^{3,11}$. Kornacki et al. reported a case of FS that showed CHAOS, a suspicion of syndactyly, and orbit asymmetry in prenatal ultrasonography; the diagnosis was confirmed by postnatal genetic analysis ${ }^{19}$. However, we did not detect these features in a subsequent fetal scan owing to severe oligohydramnios.

In conclusion, we identified novel compound heterozygous variants of FREM2 in a prenatal FS case diagnosed via ultrasonography, which is difficult but possible for fetuses exhibiting a combination of cardinal features, even if there is no affected sibling. Additionally, despite the marked interfamilial clinical heterogeneity of FS, strong phenotypic similarities exist within families ${ }^{2,20}$. Postmortem examinations of fetal and neonatal cases, followed by genetic analyses, may confirm diagnoses and consequently improve genetic counseling, prenatal molecular diagnosis, and sonographic screening during subsequent pregnancies.

\section{HGV database}

The relevant data from this Data Report are hosted at the Human Genome Variation Database at

https://doi.org/10.6084/m9.figshare.hgv.2882

https://doi.org/10.6084/m9.figshare.hgv.2885

Acknowledgements

We thank the parents for agreeing to participate in this research.

\section{Funding}

This work was supported by the Initiative on Rare and Undiagnosed Diseases (IRUD) (18ek0109301) from the Japan Agency for Medical Research and Development (AMED) (KK), the Takeda Science Foundation (KK), and the JSPS KAKENHI $18 K 07864$ (YE).

\section{Author details \\ 'Department of Obstetrics and Gynecology, Kanagawa Children's Medical Center, Yokohama, Japan. ${ }^{2}$ Clinical Research Institute, Kanagawa Children's Medical Center, Yokohama, Japan. ${ }^{3}$ Division of Medical Genetics, Kanagawa Children's Medical Center, Yokohama, Japan}

Conflict of interest

The authors declare that they have no conflict of interest.

\section{Publisher's note}

Springer Nature remains neutral with regard to jurisdictional claims in published maps and institutional affiliations.

Received: 28 June 2020 Revised: 3 August 2020 Accepted: 3 August 2020. Published online: 2 October 2020 


\section{References}

1. Thomas, I. T. et al. Isolated and syndromic cryptophthalmos. Am. J. Med. Genet. 25, 85-98 (1986).

2. Haelst, M. M., Scambler, P. J. \& Hennekam, R. C. Fraser syndrome: a clinical study of 59 cases and evaluation of diagnostic criteria. Am. J. Med. Genet. A 143A, 3194-3203 (2007)

3. Tessier, A. et al. Fraser syndrome: features suggestive of prenatal diagnosis in a review of 38 cases. Prenat. Diagn. 36, 1270-1275 (2016).

4. Bouaoud, J. et al. Fraser syndrome: review of the literature illustrated by a hystorical adult case. Int. J. Oral. Maxillofac. Surg. S0901-5027, 300007-2 (2020).

5. Martinez-Frias, M. L. et al. Fraser syndrome: frequency in our environment and clinical-epidemiological aspects of a consecutive series of cases. Esp. Pediatr. 48, 634-638 (1998).

6. McGregor, L. et al. Fraser syndrome and mouse blebbed phenotype caused by mutations in FRAS1/Fras1 encoding a putative extracellular matrix protein. Nat. Genet. 34, 203-208 (2003).

7. Pavlakis, E., Chiotaki, R. \& Chalepakis, G. The role of Tras1/Frem proteins in the structure and function of basement membrane. Int. J. Biochem. Cell Biol. 43 487-495 (2011)

8. Vogel, M. J. et al. Mutations in GRIP1 cause Fraser syndrome. J. Med. Genet. 49 303-306 (2012).

9. Murakami, H., Enomoto, Y., Tsurusaki, Y., Sugio, Y. \& Kurosawa, K. A female patient with $X$-linked Ohdo syndrome of the Maat-Kievit-Brunner phenotype caused by a novel variant of MED12. Congenit. Anom. 60, 91-93 (2020).

10. Haelst, M. M. et al. Molecular study of 33 families with Fraser syndrome new data and mutation review. Am. Med. Genet. A 146A, 2252-2257 (2008).
11. Vijayaraghavan, S. B., Suma, N., Lata, S. \& Kamakshi, K. Prenatal sonographic appearance of cryptophthalmos in Fraser syndrome. Ultrasound Obstet. Gynecol. 25, 629-630 (2005).

12. Maruotti, G. M., Paladini, D., Agangi, A. \& Martinelli, P. Prospective prenatal diagnosis of Fraser syndrome variant in a family with negative history. Prenat. Diagn. 24, 69-70 (2004).

13. Jadeja, S. et al. Identification of a new gene mutated in Fraser syndrome and mouse myelencephalic blebs. Nat. Genet. 37, 520-525 (2005).

14. Vrontou, S. et al. Fras1 deficiency results in cryptophthalmos, renal agenesis and blebbed phenotype in mice. Nat. Genet. 34, 209-214 (2003).

15. Smyth, I. \& Scambler, P. The genetics of Fraser syndrome and the blebs mouse mutants. Hum. Mol. Genet. 14, R269-R274 (2005).

16. Hedrick, M. H. et al. Congenital high airway obstruction syndrome (CHAOS): a potential for perinatal intervention. J. Pediatr. Surg. 29, 271-274 (1994).

17. Fryns, J. P., Schoubroeck, D., Vandenberghe, K., Nagels, H. M. \& Klerckx, P. Diagnostic echographic findings in cryptophthalmos syndrome (Fraser syndrome). Prenat. Diagn. 17, 582-584 (1997).

18. Messens, T., Witters, I., Robaeys, J., Peeters \& Fryns, J. P. Congenital high airway obstruction syndrome (CHAOS) as part of Fraser syndrome: Ultrasound and autopsy findings. Genet. Couns. 24, 367-371 (2013).

19. Kornacki, F., Sowinska-Sidler, A., Socha, M., Ropacka, M. \& Famsheer, A. Prenatal diagnosis of Fraser syndrome using routine ultrasound examination, confirmed by exome sequencing: report of a novel homozygous missense FRAS1 mutation. Congenit. Anom. 57, 37-38 (2017).

20. Prasun, P., Pradhan, M. \& Goel, H. Intrafamilial variability in Fraser synderome. Prenat. Diagn. 27, 778-782 (2007). 